\title{
Morphology and Mechanical Properties of Injection Molded Poly(Ethylene Terephthalate)
}

\author{
JÚLIO C. VIANA ${ }^{1,3 *}$, NATÁLIA M. ALVES ${ }^{2,3}$, and JOÃO F. MANO ${ }^{2,3}$ \\ ${ }^{1}$ IPC - Institute for Polymers and Composites \\ ${ }^{2} 3 B$ 's Research Group-Biomaterials, Biodegradables and Biomimetics \\ ${ }^{3}$ Department of Polymer Engineering \\ University of Minho \\ 4800-058, Guimarães, Portugal
}

\begin{abstract}
This work reports on the relationships between processing, the morphology and the mechanical properties of an injection molded poly(ethylene terephthalate), PET. Specimens were injection molded with different mold temperatures of $30^{\circ} \mathrm{C}, 50^{\circ} \mathrm{C}$, $80^{\circ} \mathrm{C}, 100^{\circ} \mathrm{C}, 120^{\circ} \mathrm{C}, 150^{\circ} \mathrm{C}$, while maintaining constant the other operative processing parameters. The thermomechanical environment imposed during processing was estimated by computer simulations of the mold-filling phase, which allow the calculation of two thermomechanical indices indicative of morphological development (degree of crystallinity and level of molecular orientation). The morphology of the moldings was characterized by differential scanning calorimetry (DSC) and by hot recoverable strain tests. The mechanical behavior was assessed in tensile testing at $5 \mathrm{~mm} / \mathrm{min}$ and $23^{\circ} \mathrm{C}$. A strong thermal and mechanical coupling is evidenced in the injection molding process, significantly influencing morphology development. An increase in the mold temperature induces a decrease of the level of molecular orientation (decrement in the hot recoverable strain) and an increment in the initial crystallinity of the moldings (decrement in the enthalpy of cold crystallization), also reflected in the variations of the computed thermomechanical indices. The initial modulus is mainly dependent upon the level of molecular orientation. The yield stress is influenced by both the degree of crystallinity and the level of molecular orientation of the moldings, but more significantly by the former. The strain at break was not satisfactorily linked directly to the initial morphological state because of the expected morphology changes occurring during deformation. Polym. Eng. Sci. 44:2174-2184, 2004. 우 2004 Society of Plastics Engineers.
\end{abstract}

\section{INTRODUCTION}

$\mathrm{P}$ oly(ethylene terephthalate), PET, a thermoplastic polyester widely used in packaging (e.g., containers for cosmetics, beverage bottles), is considered a highconsumption polymer of important commercial interest. PET is a slowly crystallizing polymer that can be obtained with different degrees of crystallinity $10 \%$ to $50 \%$ ) as a result of specific thermal and/or mechanical treatment to which it is submitted. Schmidt-Rohr et al. (1) suggested that the low trans conformation probability in the melt could be the principal reason for the low crystallization rate of PET. PET is also able to undergo

*To whom correspondence should be addressed. E-mail: jcv@dep.uminho.pt (C) 2004 Society of Plastics Engineers

Published online in Wiley InterScience (www.interscience.wiley.com). DOI: $10.1002 /$ pen. 20245 strain-induced crystallization when subjected to mechanical deformation $(2,3)$, for instance, during processing.

Establishing relationships between the processing and the morphological features, and between those and the mechanical properties, is of primary importance in maximizing and modeling the mechanical behavior of molded polymers. Semicrystalline polymers feature a typical layered configuration (e.g., skin-core) as a result of the high stress field and cooling rates imposed during the processing stages $(4,5)$. In the case of slowly crystallizing polymers (e.g., PET, PPS-poly(p-phenylene sulfide), PEN-poly(ethylene-2,6-naphthalate)) this layered structure may present a typical configuration: an amorphous highly oriented skin, an intermediate shear crystallized layer and an internal amorphous core (6-8). This complex morphological state reflects the importance of the shear-induced crystallization during 
injection molding of slowly crystallizing polymers. Depending on the processing conditions, these materials develop a wide variety of morphologies. For instance, if the cooling rates applied during processing are too high, they vitrify; otherwise they can crystallize. This latter ability is also enlarged by elevated stress fields. In fact, the final morphology of slowly crystallizing polymers is strongly dependent upon the cooling rate and shear stress level applied to the polymer during processing $(6,7)$. The major injection molding parameters determining the development of the microstructure of these polymers are therefore the mold temperature and injection flow rate $(6,7)$. Substantially different mechanical behaviors of the molded polymers arise as a result of the microstructures that are developed.

Amorphous polymers show no three-dimensional ordered molecular structures. For these materials, molecular orientation has been noted as an important morphological parameter influencing the mechanical response of injection moldings (9). In injection molding, the processing parameters controlling the flow-induced orientation and the relaxation phenomena should be of prime importance, such as the melt and mold temperatures and the injection flow rate. However, other morphological parameters, like entanglement density, should be also considered as affecting the mechanical response. Polymers with low degrees of crystallinity develop crystalline entities, which are embedded in an amorphous matrix. It has been shown that the mobility of the amorphous matrix of PET is greatly affected by the proximity of the crystalline phase $(10-12)$. In fact, when the amount of crystalline phase is small, two amorphous phases with differentiated conformational mobility and consequently two distinct glass transition processes can be observed $(13,14)$. Furthermore, molded semicrystalline polymers feature a layered structure as a result of the thermal and mechanical conditions imposed during processing (e.g., extrusion, injection molding). Some morphological parameters have been identified as determinants of the mechanical behavior of these materials, such as the skin ratio, the level of molecular orientation of the skin and the crystalline structure of the core $(5,15-18)$. However, the different mechanical properties (e.g., initial modulus, yield stress, toughness) are determined by distinct morphological features $(18,19)$.

This work studies the relationships between the morphology and the mechanical behavior of injection molded PET. Specifically, the mold temperature was varied in a wide range covering the glass transition temperature of the material $\left(T_{g} \approx 70^{\circ} \mathrm{C}\right)$. This is expected to have a great effect upon the crystallization kinetics of this slowly crystallizing polymer (6-8), controlling the development of the morphology and therefore determining the mechanical behavior of the moldings. This study is part of a broader program that intends to understand the relationships between processing, the morphology development and the mechanical behavior of thermoplastic materials. Future studies will consider other processing variables in order to analyze their relative contribution in the final microstructure and properties.

\section{EXPERIMENTAL}

\section{Material and Molding}

The material was a copolymer of PET and isophthalic acid typically used in the packaging industry (grade S86G from Selenis: intrinsic viscosity of $0.81 \pm 0.02$ $\mathrm{dl} / \mathrm{g})$. The molding is a dumbbell tensile specimen 60 $\mathrm{mm}$ long, with a constant rectangular cross section of $4 \times 2 \mathrm{~mm}$, and $20 \mathrm{~mm}$ of reference length.

The PET was dried before processing at $100^{\circ} \mathrm{C}$ for 16 hours (dry air with a dew point of $-40^{\circ} \mathrm{C}$ ). The specimens were then injection molded at different mold temperatures of $30^{\circ} \mathrm{C}, 50^{\circ} \mathrm{C}, 80^{\circ} \mathrm{C}, 100^{\circ} \mathrm{C}, 120^{\circ} \mathrm{C}$, and $150^{\circ} \mathrm{C}$. The temperature control fluid was water for the first three mold temperatures and oil for the others. The surface mold temperature was verified for each run, with values equal to the setting points of the temperature controllers. The other operative molding parameters were kept constant: melt temperature at $270^{\circ} \mathrm{C}$, flow rate at $50 \mathrm{~cm}^{3} \cdot \mathrm{s}^{-1}$, and holding pressure at $20 \mathrm{MPa}$ (at the screw tip).

\section{Thermomechanical Environment}

The thermomechanical environment imposed on the polymer during injection molding was estimated by computer simulations of the mold-filling phase. The simulations were performed in the C-Mold package. The material was assumed to be a temperature-dependent shear-thinning fluid. The processing conditions replicated those set in the experimental injection molding program. The thermomechanical variables computed from the simulations were the bulk temperature, $T_{b}$, and the shear stress at the solid-melt interface, $\tau_{w}$, both taken at the middle length of the specimens. Both these values were assessed at the end of filling. Microstructural development was interpreted by means of two thermomechanical indices (19-21): the cooling index, $Y_{c}$, and the thermo-stress index, $\tau_{Y}$ :

$$
\begin{gathered}
Y_{c}=\frac{T_{b}-T_{t}}{T_{b}-T_{i}} \\
\tau_{Y}=\frac{\tau_{w}}{Y_{c}}
\end{gathered}
$$

where $T_{t}$ is the polymer transition temperature (crystallization temperature for semicrystalline polymers and glass transition temperature for amorphous materials; for PET a constant value of $T_{t}=T_{g}=70^{\circ} \mathrm{C}$ was used) and $T_{i}$ is the mold/polymer interface temperature defined by (22):

$$
T_{i}=\frac{b_{p} T_{b}+b_{w} T_{w}}{b_{p}+b_{w}}
$$

where $T_{w}$ is the mold temperature, $b$ is the thermal effusivity $\left(b=\sqrt{\rho C_{p} K} ; \rho\right.$ is the density, $C_{p}$ is the specific 
heat capacity and $K$ the thermal conductivity) and the subscripts " $p$ " and " $w$ " stand for polymer and mold, respectively $\left(b_{p}=6.93 \times 10^{2} \mathrm{~J} \cdot{ }^{\circ} \mathrm{C}^{-1} \cdot \mathrm{m}^{-2} \cdot \mathrm{s}^{-1 / 2}\right.$ and $b_{w}=$ $\left.11.46 \times 10^{3} \mathrm{~J} \cdot{ }^{\circ} \mathrm{C}^{-1} \cdot \mathrm{m}^{-2} \cdot \mathrm{s}^{-1 / 2}\right) . Y_{c}$ evaluates the thermal level of the molding, in the case of semicrystalline polymers being related to their degree of crystallinity $(20,21), \tau_{Y}$ is proportional to the level of molecular orientation of the molding $(20,21)$. These indices have never been applied to interpret the morphology development in injection molding of low degree of crystallinity or amorphous materials.

\section{Morphological Characterization}

\section{$X$-ray Scattering Studies}

Some of the moldings $\left(T_{w}=30^{\circ} \mathrm{C}, 80^{\circ} \mathrm{C}\right.$ and $\left.150^{\circ} \mathrm{C}\right)$ were characterized by wide-angle X-ray (WAXS) measurements. These experiments were performed under synchrotron radiation at HASYLAB, DESY, Hamburg (A2 soft condensed matter beam-line). The specimens were positioned perpendicular to the incident X-ray beam with the flow direction pointing upward. The exposure time was $3 \mathrm{~s}$. The two-dimensional WAXS patterns were acquired by a MARCCD camera, allowing us to obtain the equatorial plots. Background scattering was subtracted, and all plots were normalized with respect to the incident X-ray intensity and initial specimen thickness.

\section{Differential Scanning Calorimetry}

The morphological characterization was performed by differential scanning calorimetry (DSC) in bulk specimens cut from the center of the tensile specimens. All the experiments were conducted in a PerkinElmer DSC-7 differential scanning calorimeter with a controlled cooling accessory and in a nitrogen atmosphere. Two samples per condition, with weight of 6-7 mg, were used. The samples were heated from $30^{\circ} \mathrm{C}$ to $275^{\circ} \mathrm{C}$ at a heating rate of $10^{\circ} \mathrm{C} / \mathrm{min}$. Prior to the experiments, the temperature of the equipment was calibrated with indium and lead standards and only the same indium sample was used for the heat flow calibration. The calibrations were performed at the same heating rate of the experiments. The cold crystallization enthalpy, $\Delta H_{c}$, and the melting enthalpy, $\Delta H_{m}$, were calculated from the thermograms. The glass transition, the cold crystallization and the melting peak temperatures were also identified, respectively, $T_{g}, T_{c}$ and $T_{m}$.

\section{Hot Recoverable Strain Tests}

The level of amorphous molecular orientation resulting from processing can relax upon thermal treatment at a temperature slightly higher than the $T_{g}$. This results in variations on the sample dimensions. The difference between the dimension before and after the thermal treatment is called the hot recoverable strain, $\varepsilon_{\mathrm{HR}}$. The higher the $\varepsilon_{\mathrm{HR}}$ (that is, the more the material recovers), the higher is expected to be the initial level of molecular orientation (23).

Four specimens of each condition were marked with a grid spaced by $5.0 \mathrm{~mm}$ and $20 \mathrm{~mm}$ long along the constant cross section. The specimens were then placed over a fine talc layer in an oven with a controlled temperature of $80^{\circ} \mathrm{C}$ for $10 \mathrm{~min}$, after which the oven was turned off and the specimens allowed to cool for 24 hours. The grid marks were remeasured in each specimen. The hot recoverable strain, $\varepsilon_{\mathrm{HR}}$, is defined as the average percentage reduction on the distance between the grid marks. The higher the $\varepsilon_{\mathrm{HR}}$ values, the higher the expected level of molecular orientation in the flow direction. The experimental scatter on the results was estimated to be, on average, $12 \%$.

\section{Mechanical Characterization}

The dumbbell-like specimens were tested in a computerized Universal testing machine, Instron 4505, in tensile mode. The tests were performed at controlled room temperature of $23^{\circ} \mathrm{C}$ at a test velocity of $8.33 \times$ $10^{-4} \mathrm{~m} . \mathrm{s}^{-1}$ (5 mm. $\mathrm{min}^{-1}$, corresponding to a nominal strain rate of $4.2 \times 10^{-3} \mathrm{~s}^{-1}$ ). At least four specimens for each condition were tested. The mechanical properties envisaged were the initial modulus, E; the nominal yield stress, $\sigma_{\mathrm{y}}$; and the nominal strain at break, $\varepsilon_{\mathrm{b}}$. The experimental scatters on the measurements were $4.2 \%, 2.5 \%$ and $17.3 \%$, respectively, for $\mathrm{E}, \sigma_{\mathrm{y}}$. and $\varepsilon_{\mathrm{b}}$.

\section{RESULTS}

\section{Molding and Thermomechanical Environment}

Figure 1 depicts the appearance of the injection molded tensile specimens as observed between cross polarizers. As $T_{w}$ increases, the samples become less transparent, presaging the occurrence of some crystallization during processing, and leading to a more opaque appearance of the moldings. An exception to this trend occurs for the sample molded with $T_{w}=$ $100^{\circ} \mathrm{C}$, which presents an unexpectedly low level of opacity and almost no birefringence. This is a consequence of the particular thermomechanical conditions applied during processing, which seem to lead to a low level of molecular orientation and little crystallization of the molding.

Table 1 summarizes the values of the thermomechanical variables and indices (Eqs 1 and 2) computed from the mold filling simulations as function of $T_{w}$. The thermomechanical variables, $T_{b}$ and $\tau_{w}$, change only slightly with the mold temperature. However, this is not the case for the thermomechanical indices, $Y_{c}$ and $\tau_{Y}$, which vary by $94.3 \%$ and $98.6 \%$, respectively, presaging major differences in the morphological state of the moldings. Figure 2 shows the evolution of $Y_{c}$ and $\tau_{Y}$ with $T_{w}$. As expected, an increment in $T_{w}$ results in an increase of $Y_{c}$, that is, of the thermal level of the moldings, which presages a presumably higher degree of crystallinity of the moldings. The increase of $T_{w}$ leads also to lower $\tau_{Y}$ values, reflecting the eventual inferior level of molecular orientation of the molded samples. Further, and as already reported (18-20), a strong thermal and mechanical coupling is evidenced by the fact that higher a $Y_{c}$ corresponds to a lower $\tau_{Y}$, and vice versa. 
Fig. 1. Samples injection molded with different mold temperatures observed between cross polarizers. [Color figure can be viewed in the online issue, which is available at www.interscience.wiley.com.]

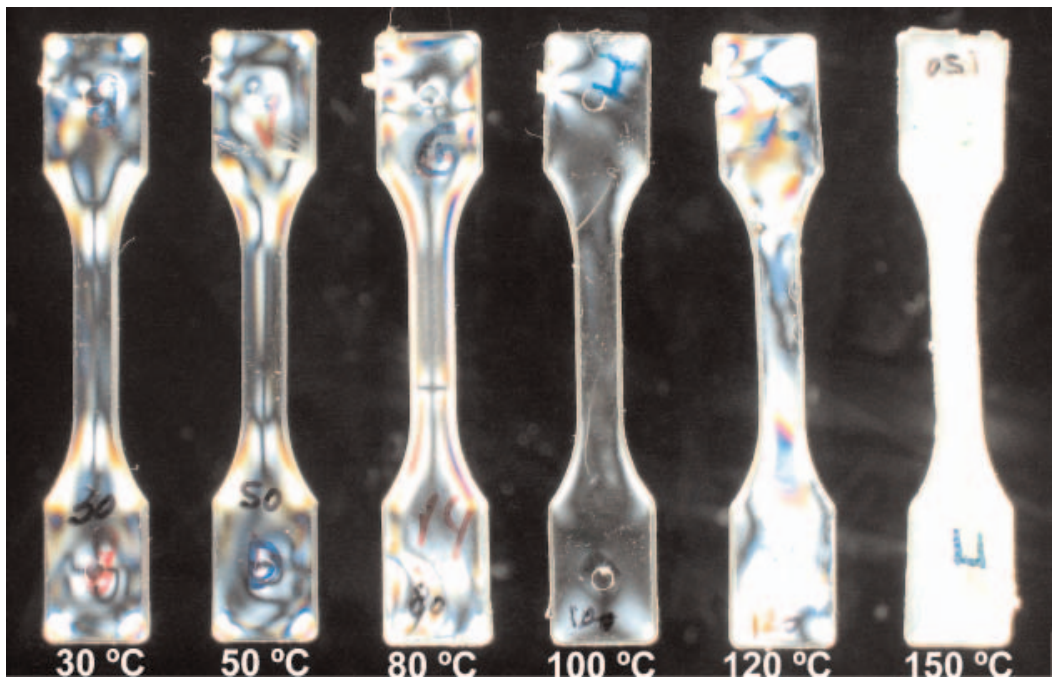

Table 1. Characterization of the Thermomechanical Environment by Mold Filling Simulations.

\begin{tabular}{ccccc}
\hline $\boldsymbol{T}_{\boldsymbol{w}}\left({ }^{\circ} \mathbf{C}\right)$ & $\boldsymbol{T}_{\boldsymbol{b}}\left({ }^{\circ} \mathbf{C}\right)$ & $\boldsymbol{\tau}_{w}(\mathrm{MPa})$ & $\boldsymbol{Y}_{\boldsymbol{c}}$ & $\boldsymbol{\tau}_{Y}(\mathrm{MPa})$ \\
\hline 30 & 276.2 & 0.582 & 0.845 & 0.689 \\
50 & 276.4 & 0.577 & 0.920 & 0.627 \\
80 & 276.8 & 0.570 & 1.061 & 0.538 \\
100 & 277.0 & 0.568 & 1.180 & 0.481 \\
120 & 277.3 & 0.567 & 1.330 & 0.426 \\
150 & 277.7 & 0.570 & 1.642 & 0.347 \\
$\operatorname{Var}(\%)$ & 0.4 & 2.7 & 94.3 & 98.6 \\
\hline
\end{tabular}

( $T_{b}$-bulk temperature, $\tau_{w}$-wall shear stress, $Y_{c}$-cooling index $(E q 1), \tau_{Y}$-thermo-stress index (Eq 2); Var-percentage of variation [(max - min)/min].)

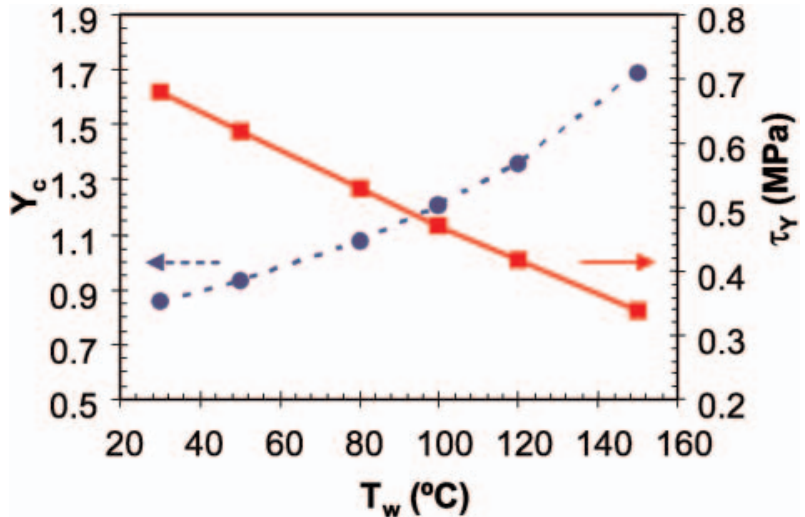

Fig. 2. Variations of the cooling index, $Y_{c}$, and thermo-stress index, $\tau_{Y}$, with the mold temperature, $T_{w}$. [Color figure can be viewed in the online issue, which is available at www. interscience.wiley.com.]

\section{Morphological Characterization}

\section{WAXS Characterization}

Figure 3 shows the intensity vs. $2 \theta$ curves and respective two-dimensional WAXS patterns for moldings processed at $T_{w}=30^{\circ} \mathrm{C}, 80^{\circ} \mathrm{C}$ and $150^{\circ} \mathrm{C}$. The $2 \mathrm{D}$-WAXS images reveal the very low degree of crystallinity of the specimens that feature a well-defined amorphous halo. It has been suggested that PET may evidence a precrystalline state, which is neither crystalline nor fully

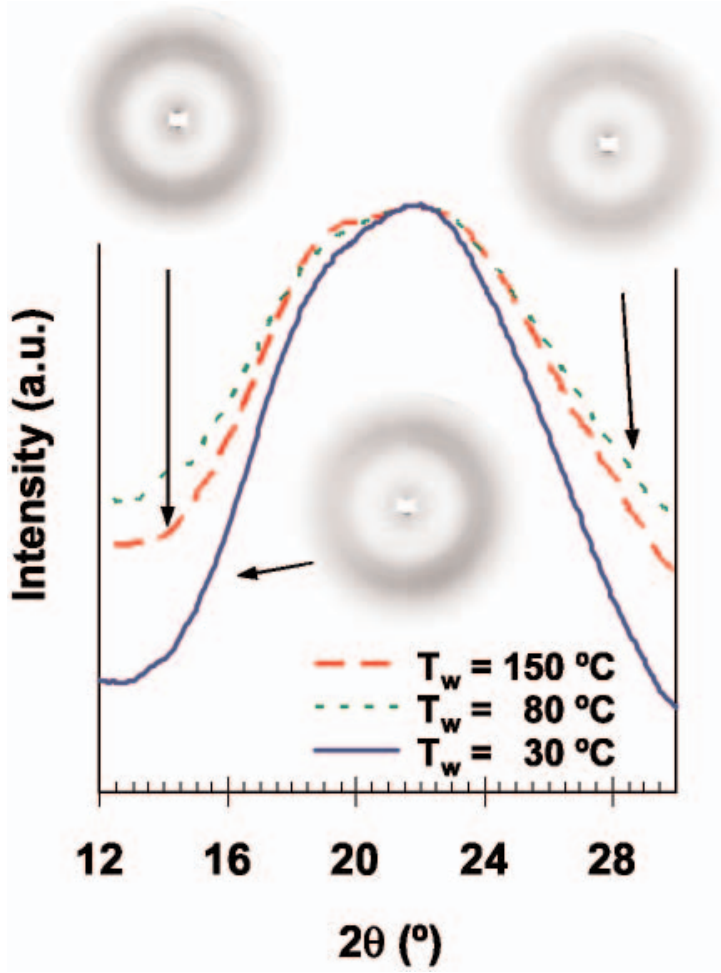

Fig. 3. Intensity versus $2 \theta$ curves and correspondent WAXS $2 D$ patterns for selected moldings $\left(T_{w}=30^{\circ} \mathrm{C}, 80^{\circ} \mathrm{C}\right.$ and $\left.150^{\circ} \mathrm{C}\right)$. [Color figure can be viewed in the online issue, which is available at www.interscience.wiley.com.] 
amorphous. This state is characterized by the absence of crystalline reflections in the WAXS pattern, by the occurrence of a SAXS maximum and by an exothermic peak in DSC scan (24). Nevertheless, as $T_{w}$ increases, a very weak crystalline reflection starts to appear. In the intensity vs. $2 \theta$ curves of Fig. 3, the y-axis has arbitrary units and the curves are vertically shifted so that all coincide at the maximum peak. For $T_{w}=30^{\circ} \mathrm{C}$, no crystalline peak can be distinguished. For $T_{w}=150^{\circ} \mathrm{C}$, a very weak crystalline peak appears at $2 \theta \approx 19^{\circ} \mathrm{C}$, indicating a slightly higher degree of crystallinity of this molding.

\section{Differential Scanning Calorimetry}

Figure 4 presents the DSC thermograms of the samples molded with the different mold temperatures. The different initial morphological state of the samples is mainly evidenced in the cold crystallization peak. The area of these peaks decreases with increasing $T_{w}$, indicating an increment in the initial crystallinity of the moldings. Further, the peak temperature is moved to higher temperatures as $T_{w}$ increases as a consequence of slower crystallization kinetics. This may be attributed to the lower level of molecular orientation of the moldings (25). In fact, a decrease in the crystallization rate with increasing cooling rate during sample preparation was observed for non-oriented PET samples, which was attributed to a reduction of the nucleation density (24), as opposed to our initially highly oriented samples.

Table 2 lists the DSC results. $T_{g}$ is not affected by the changes in the mold temperature. $T_{g}$ was found to increase, and the transition magnitude to decrease, with increasing degrees of crystallinity because the crystalline entities geometrically constrain the amorphous phase, inhibiting the segmental motions (26). In this context, a more precise treatment was performed where DSC results for PET with different degrees of crystallinity were analyzed with a model based on the configurational entropy concept (14). On the other hand, a reduction in the molecular orientation increases the free volume within the amorphous fraction and allows for more ample movements of the amorphous regions, decreasing $T_{g}$ (27). Either these opposed effects or their low magnitude may contribute to the insensitivity of processing to variations of $T_{g}$.

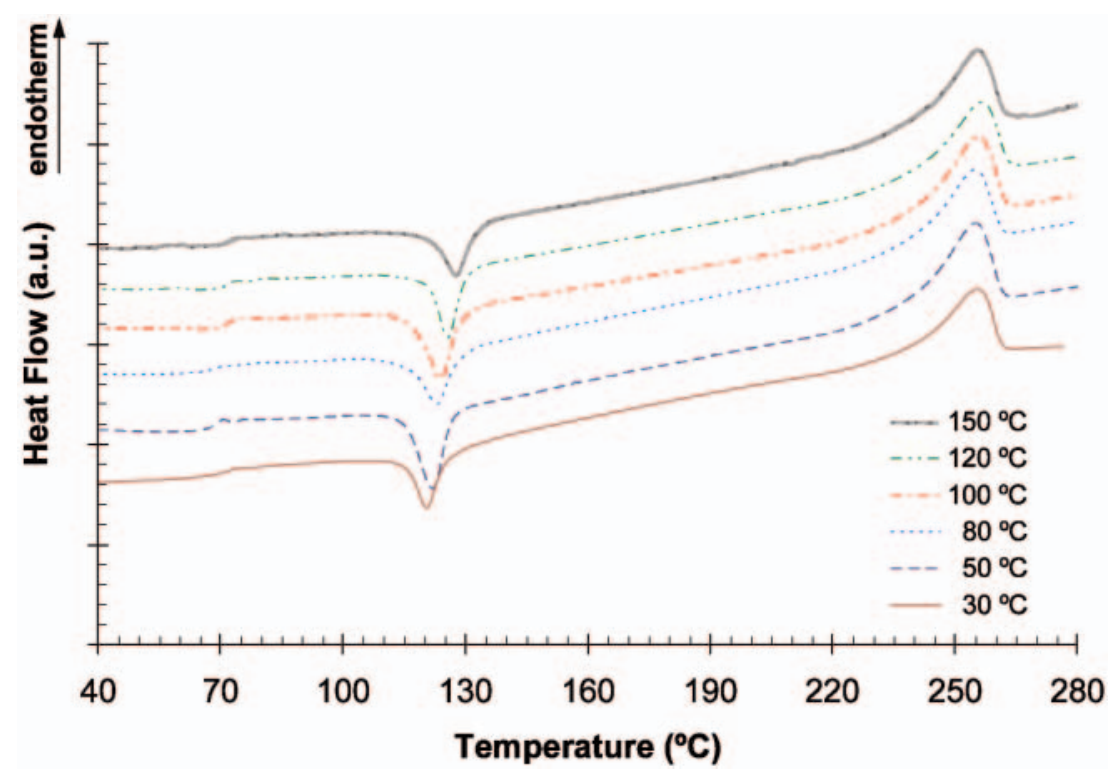

Fig. 4. DSC thermograms at $10^{\circ} \mathrm{C} / \mathrm{min}$ for the PET samples molded with different mold temperatures, $T_{w}$. [Color figure can be viewed in the online issue, which is available at www.interscience.wiley.com.]

Table 2. Results From the DSC and Hot Recoverable Strain Tests (Standard Deviations Are in Parentheses).

\begin{tabular}{cccccccc}
\hline $\boldsymbol{T}_{\boldsymbol{w}}\left({ }^{\circ} \mathbf{C}\right)$ & $\boldsymbol{T}_{\boldsymbol{g}}\left({ }^{\circ} \mathbf{C}\right)$ & $\boldsymbol{T}_{\boldsymbol{c}}\left({ }^{\circ} \mathbf{C}\right)$ & $\Delta \boldsymbol{H}_{\boldsymbol{c}}(\mathbf{J} / \mathbf{g})$ & $\boldsymbol{T}_{\boldsymbol{m}}\left({ }^{\circ} \mathbf{C}\right)$ & $\Delta \boldsymbol{H}_{\boldsymbol{m}}(\mathrm{J} / \mathbf{g})$ & $\chi_{\mathrm{m}}(\%)$ & $\boldsymbol{\varepsilon}_{\mathrm{HR}}(\%)$ \\
\hline 30 & $71.0 \pm 0.5$ & $120.5 \pm 0.8$ & $25.9 \pm 1.1$ & $255.1 \pm 0.1$ & $51.7 \pm 2.2$ & 21.5 & $4.07(0.44)$ \\
50 & $72.4 \pm 2.0$ & $122.8 \pm 0.8$ & $27.5 \pm 2.5$ & $254.8 \pm 0.2$ & $55.0 \pm 2.4$ & 22.9 & $3.41(0.01)$ \\
80 & $69.9 \pm 1.3$ & $123.7 \pm 0.6$ & $26.4 \pm 1.8$ & $254.0 \pm 0.5$ & $55.9 \pm 0.1$ & 24.6 & $3.08(0.07)$ \\
100 & $71.1 \pm 0.5$ & $124.8 \pm 0.9$ & $24.8 \pm 2.0$ & $254.8 \pm 0.6$ & $49.5 \pm 0.3$ & 20.6 & $2.31(0.46)$ \\
120 & $72.5 \pm 0.4$ & $125.8 \pm 0.2$ & $22.1 \pm 0.6$ & $255.8 \pm 0.6$ & $47.9 \pm 2.6$ & 21.5 & $1.40(0.03)$ \\
150 & $72.0 \pm 0.2$ & $127.2 \pm 0.4$ & $21.6 \pm 0.3$ & $255.2 \pm 0.1$ & $51.9 \pm 1.4$ & 25.2 & $1.31(0.47)$ \\
$\operatorname{Var}(\%)$ & 3.7 & 5.6 & 27.2 & 0.7 & 16.8 & 22.2 & 210.5 \\
\hline
\end{tabular}

( $T_{w}$-mold temperature, $T_{c}$-cold crystallization peak temperature, $\Delta H_{c}$-enthalpy of cold crystallization, $T_{m}$-melting peak temperature, $\Delta H_{m}-$ enthalpy of melting, $\chi_{m}-$ degree of crystallinity calculated as $\left[\left(\Delta H_{m}-\Delta H_{c}\right) / \Delta H_{f}\right]\left(\Delta H_{f}=120 \mathrm{~J} / \mathrm{kg}\right.$ from (2)), $\varepsilon_{\mathrm{HR}}$ - hot recoverable strain; Var-percentage of variation [(max - min)/min].) 
Determining the degree of crystallinity of PET by DSC is not a trivial task. It could be calculated from the difference between the melting and cold crystallization enthalpies $(3,28)$, but this is only an indicative value and its application to highly oriented samples can be questionable (these values are listed in Table 2; the initial degree of crystallinity of the PET moldings varies from $20 \%$ to $25 \%$ ). The melting peak corresponds to the melting of the portion of material that has crystallized under the thermomechanical environment applied during processing and those that have crystallized during the heating run but under the constraints imposed by the morphology previously developed upon processing. In fact, the crystallization kinetics of PET are affected by the level of molecular orientation of the material (29). The interpretation of the melting peak therefore appears to be of a complex nature, and its variation will not be discussed further here. However, it is true that the less crystalline the initial PET material (resulting from processing), the more it crystallizes upon heating and a more pronounced cold crystallization peak with a higher $\Delta H_{c}$ value is observed. Then, it can be said that $\Delta H_{c}$ is directly related to the initial crystallinity of the samples. So, the results of Table 2 seem to indicate that the higher the $T_{w}$, the more crystalline the material (low $\Delta H_{c}$ ). The variations of $\Delta H_{c}$ and $T_{c}$ with the mold temperature are presented in Fig. 5. $\Delta H_{c}$ decreases as the mold temperature increases, indicating an increment of the degree of crystallinity of the moldings. A simple analysis revealed a quasi-quadratic dependence between $\Delta H_{c}$ and $T_{w}$ (dotted line in Fig. 5), with a coefficient of correlation $R^{2}=0.85$. Note that such correlations are purely empirical, and no further discussion about the best functional relationship will be given here. The variations of $T_{c}$ with $T_{w}$ are not very pronounced (5.6\%), but a linear relationship (solid line) seems to exist with a coefficient of correlation of $R^{2}=$ 0.97. The slower crystallization kinetics shown by the samples molded with the highest $T_{w}$ is the result of its initial state, namely its higher degree of crystallinity and eventual lower level of molecular orientation. In fact, an oriented amorphous phase serves as a precursor for

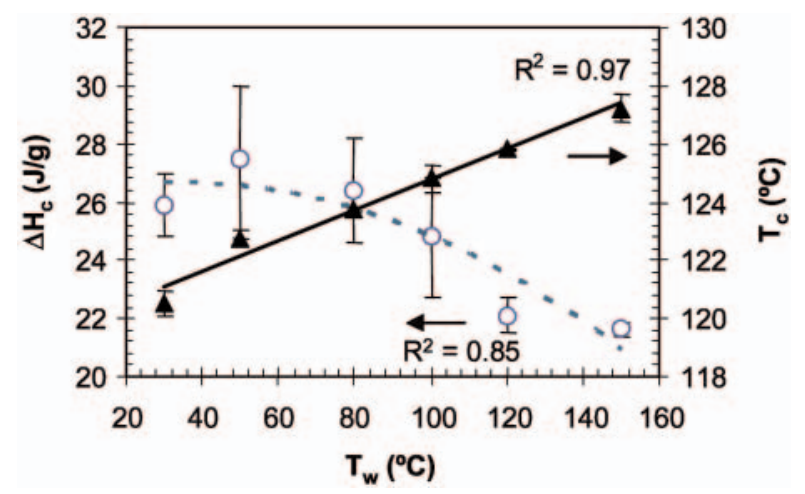

Fig. 5. Variations of the enthalpy of cold crystallization, $\Delta H_{c}$, and peak temperature, $T_{c}$, with the mold temperature, $T_{w}$. [Color figure can be viewed in the online issue, which is available at www.interscience.wiley.com.] extended chain crystallization (30), so samples with higher orientation will crystallize first, in this case, at lower temperatures (25). As the molecular orientation, as expected, decreases with increasing $T_{w}, T_{c}$ will also increase as $T_{w}$ increases. The mold temperature seems to have a strong influence on the morphological state of the moldings of slowly crystallizing polymers. $T_{w}$ has also been shown to be an important processing parameter in determining the crystallization kinetics of rapidcrystallizing polymers (such as polypropylene) $(21,31)$.

\section{Hot Recoverable Strain Tests}

Table 2 also lists the results of the hot recoverable tests. $\varepsilon_{\mathrm{HR}}$ decreases with $T_{w}$, being one of the parameters with the highest variation (of more than 200\%). This suggests the strong effect of $T_{w}$ on the level of molecular (amorphous) orientation of the material. Figure 6 depicts the dependence of the hot recoverable strain, $\varepsilon_{\mathrm{HR}}$, upon $T_{w}$. The higher the level of molecular orientation of the molding, the higher is the recovery upon heating. The diminution of $\varepsilon_{\mathrm{HR}}$ with $T_{w}$ is related to a significant decrease of the global level of molecular orientation of the moldings. The variation is linear, as shown by the solid line in Fig. 6 (coefficient of correlation of $\left.R^{2}=0.95\right)$. For the case of rapid-crystallizing polymers, the influence of the mold temperature on the level of crystalline phase orientation (measured by wide-angle X-ray diffraction) was found to be negligible $(21,31)$.

\section{Mechanical Characterization}

Figure 7 presents the engineering stress-strain curves for the molding obtained with the distinct mold temperatures. The influence of $T_{w}$ on the deformation capabilities of the moldings is clearly evidenced by the distinct strain at break shown by the moldings. Furthermore, the high strain level (higher than 240\%) behavior is independent of the initial morphological state of the moldings as induced by variations in the mold temperature. The assessed mechanical properties are listed in Table 3. As already observed, $T_{w}$ has a great effect upon

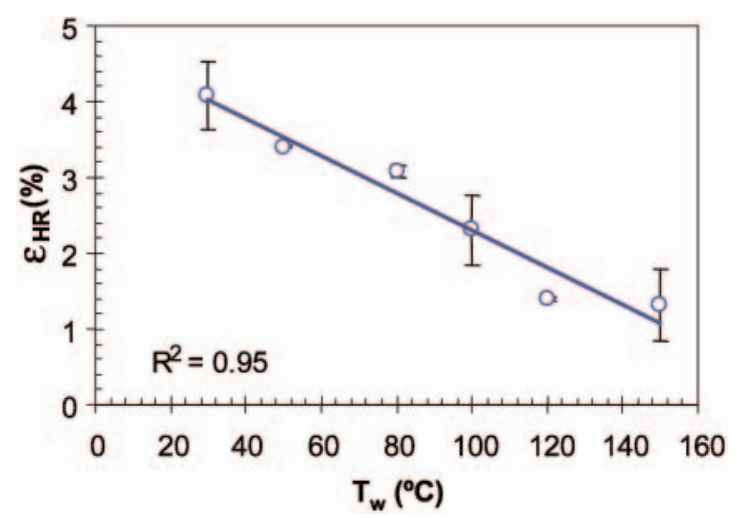

Fig. 6. Variation of the hot recoverable strain, $\varepsilon_{H R}$, with the mold temperature, $T_{w}$. [Color figure can be viewed in the online issue, which is available at www.interscience.wiley.com.] 


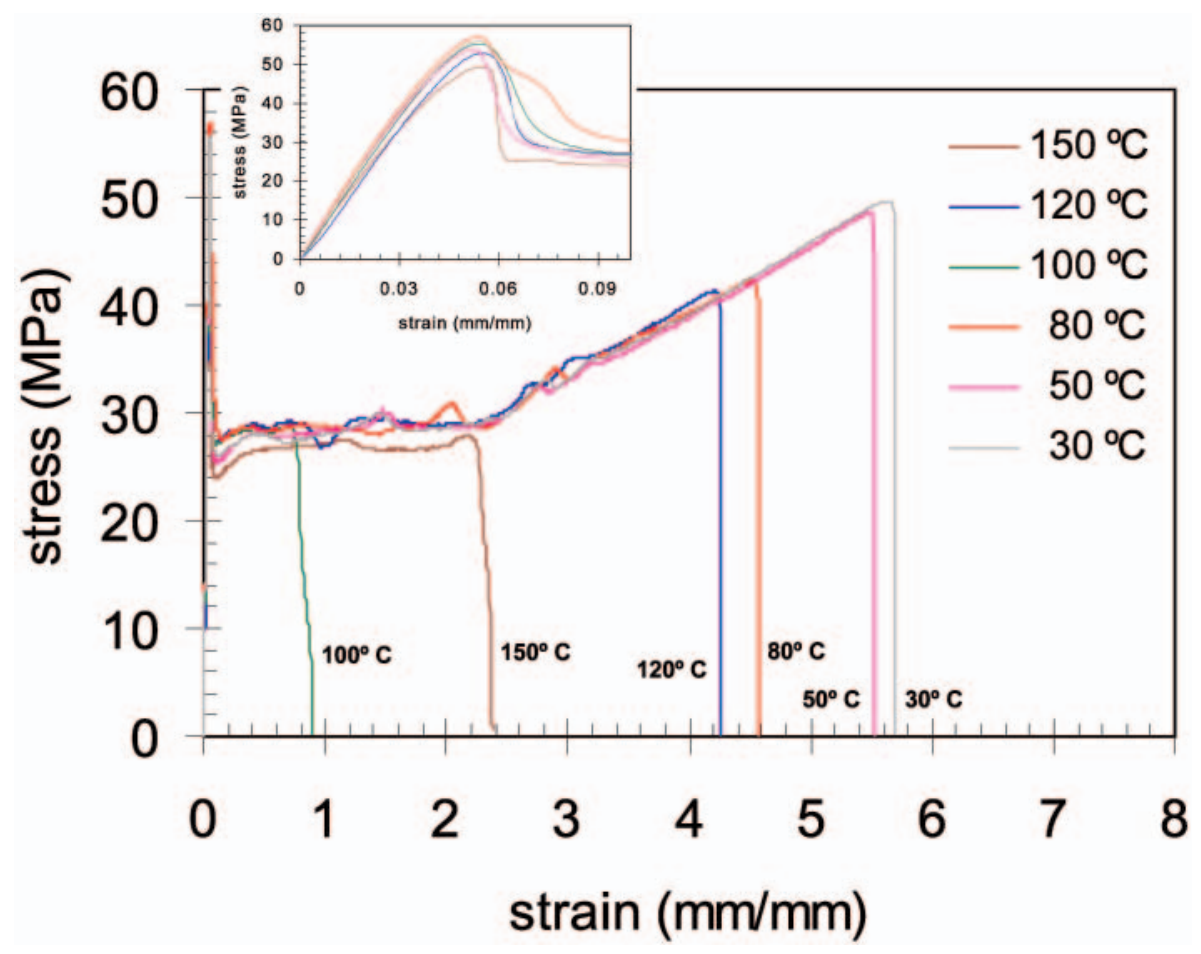

Fig. 7. Experimental engineering stress-strain curves for different mold temperatures. [Color figure can be viewed in the online issue, which is available at www.interscience.wiley.com.]

the strain and energy at break (variations of $478 \%$ and $604 \%$, respectively). The initial modulus and the yield stress also vary slightly with $T_{w}$ (respectively, 15\% and $11 \%$ ), but the yield strain is kept constant. In all cases, the variations in the mechanical properties are not monotonic with $T_{w}$. This indicates the different contributions of the distinct morphological parameters to the mechanical properties, as the result of changing $T_{w}$.

The dependences of the mechanical properties upon the mold temperature are depicted in Fig. 8 for the initial modulus and yield stress (respectively, E and $\sigma_{\mathrm{y}}$ ) and for the strain and energy at break $\left(\varepsilon_{\mathrm{b}}\right.$ and $\mathrm{U}_{\mathrm{b}}$, respectively) in Fig. 9. When the mold temperature ranges between $30^{\circ} \mathrm{C}$ and $80^{\circ} \mathrm{C}, \mathrm{E}$ is independent of $T_{w}$ (Fig. 8). From $T_{w}=80^{\circ} \mathrm{C}$ until $120^{\circ} \mathrm{C}, \mathrm{E}$ decreases with $T_{w}$. At this latter $T_{w}$ value, $\mathrm{E}$ shows its lowest value. Thereafter it starts to increase (up to $T_{w}=150^{\circ} \mathrm{C}$ ). $\sigma_{\mathrm{y}}$ shows a similar trend of variations but within different temperature limits: from $T_{w}=30^{\circ} \mathrm{C}$ to $50^{\circ} \mathrm{C}, \sigma_{\mathrm{y}}$ decreases with $T_{w}$, and begins to increase from then on (until reaching a threshold value at $T_{w}=100^{\circ} \mathrm{C}$ ). A more complex behavior is shown in Fig. 9 for the dependence of the strain and energy at break with $T_{w}$. A minimum at $T_{w}$ $=100^{\circ} \mathrm{C}$ is shown for both ultimate properties, indicating the lowest energy absorption capabilities of the moldings. It is interesting to note that this sample was also evidently an exception based on its optical appearance between cross polarizers (Fig. 1).

The trends shown in Figs. 7 to 9 should be related to the distinct influence of $T_{w}$ on the morphological parameters and to those on the mechanical response.

\section{DISCUSSION}

\section{Processing-Morphology Relationships}

The approach followed so far of directly linking the processing conditions (machine adjusted) to the morphological parameters is not satisfactory (Figs. 5 and

Table 3. Results From the Tensile Tests at $8.33 \times 10^{-4} \mathrm{~m} \cdot \mathrm{s}^{-1}\left(5 \mathrm{~mm} \cdot \mathrm{min}^{-1}\right)$ and $23^{\circ} \mathrm{C}$ (Standard Deviations in Parentheses).

\begin{tabular}{cccrrr}
\hline $\boldsymbol{T}_{\boldsymbol{w}}\left({ }^{\circ} \mathbf{C}\right)$ & $\mathrm{E}(\mathrm{MPa})$ & $\boldsymbol{\sigma}_{\mathbf{y}}(\mathrm{MPa})$ & $\boldsymbol{\varepsilon}_{\mathbf{y}}(\mathrm{mm} / \mathbf{m m})$ & \multicolumn{1}{c}{$\boldsymbol{\varepsilon}_{\mathbf{b}}(\mathrm{mm} / \mathbf{m m})$} & $\mathbf{U}_{\mathbf{b}}(\mathrm{J})$ \\
\hline 30 & $1353(37)$ & $56.5(0.93)$ & $1.65(0.04)$ & $5.57(0.06)$ & $31.69(0.07)$ \\
50 & $1334(52)$ & $51.2(1.02)$ & $1.99(0.03)$ & $5.67(0.25)$ & $32.5(2.81)$ \\
80 & $1373(47)$ & $53.1(0.78)$ & $1.46(0.02)$ & $4.3865(0.05)$ & $23.1(0.04)$ \\
100 & $1269(76)$ & $56.5(0.50)$ & $0.89(0.03)$ & $0.9815(0.70)$ & $4.62(3.33)$ \\
120 & $1187(71)$ & $56.4(1.34)$ & $2.34(0.03)$ & $4.295(0.54)$ & $22.11(3.23)$ \\
150 & $1294(34)$ & $56.8(2.10)$ & $6.91(0.03)$ & $1.964(0.25)$ & $8.4(1.19)$ \\
$\operatorname{Var}(\%)$ & 15.7 & 10.3 & 2.5 & 477.7 & 603.5 \\
\hline
\end{tabular}

$\left(T_{w}\right.$-mold temperature, E-initial modulus, $\sigma_{y}$-yield stress, $\varepsilon_{y}$-yield strain, $\varepsilon_{b}$-strain at break, $\mathrm{U}_{\mathrm{b}}$-energy at break; Var-percentage of variation $\left.[(\mathrm{max}-\mathrm{min}) / \mathrm{min}].\right)$ 


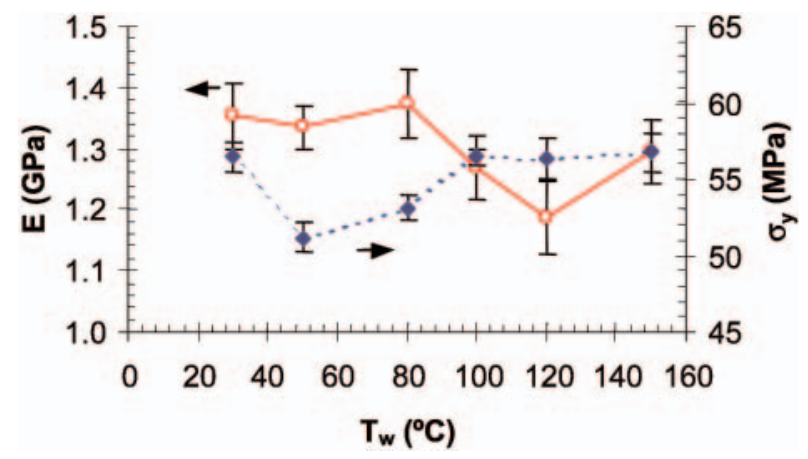

Fig. 8. Variations of the initial modulus, E (solid line) and yield stress, $\sigma_{y}$ (broken line), with the mold temperature, $T_{w}$. [Color figure can be viewed in the online issue, which is available at www.interscience.wiley.com.]

6). The morphology development is controlled by the thermomechanical environment imposed on the material inside the mold (that is, during the filling, packing and cooling phases of the injection molding cycle). The definition of the thermomechanical indices (Eqs 1 and 2) aimed at establishing this connection.

Figure 10 depicts the evolution of the cold crystallization enthalpy and peak temperature with the cooling index, $Y_{c} . \Delta H_{c}$ decreases (and the degree of crystallinity of the moldings increase) with $Y_{c}$. This type of direct relationship between $Y_{c}$ and the degree of crystallinity of the moldings was previously reported for rapid-crystallizing polymers (PP and PE $(20,21))$.

The variations in Fig. 10 are similar to those shown in Fig. 5. In our case, $Y_{c}$ is essentially determined by $T_{w}$, as shown in Fig. 2. $T_{w}$ is the processing parameter controlling the cooling rate of the material, and therefore with higher influence on the degree of crystallinity of the molding $(20,21,31)$. The cooling index, $Y_{c}$, is related to the degree of crystallinity of the moldings and to the thickness of lamella, as previously reported elsewhere for rapid crystallizing polymers (19-21).

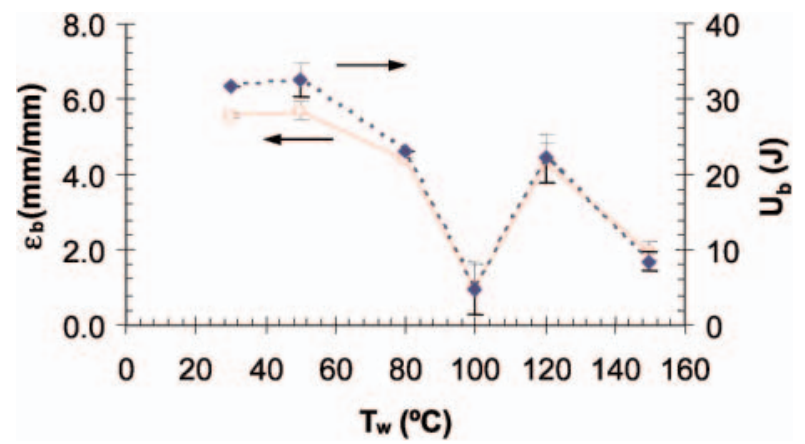

Fig. 9. Variations of the strain and energy at break, $\varepsilon_{b}$ (solid line) and $U_{b}$, (broken line) with the mold temperature, $T_{w}$. [Color figure can be viewed in the online issue, which is available at www.interscience.wiley.com.]

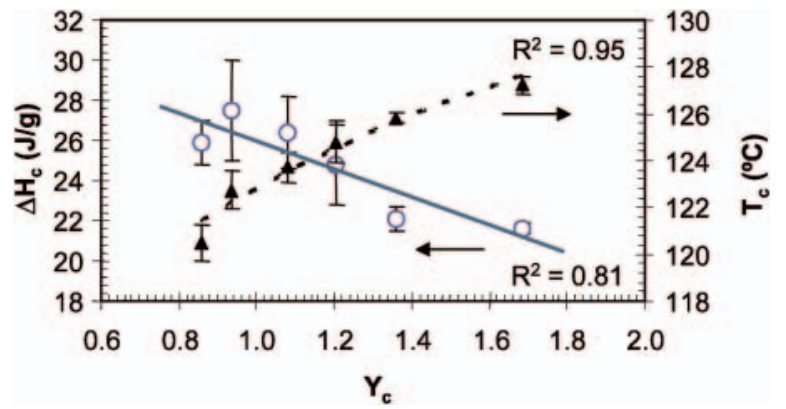

Fig. 10. Variations of the enthalpy of cold crystallization, $\Delta H_{c}$, and peak temperature, $T_{c}$, with the cooling index, $Y_{c^{*}}$ [Color figure can be viewed in the online issue, which is available at www.interscience.wiley.com.]

Figure 11 shows the variation of the hot recoverable strain, $\varepsilon_{\mathrm{HR}}$, with the thermo-stress index, $\tau_{Y}$. Both quantities are proportionally related, as already reported (19-21). From Figs. 10 and 11, one may conclude that the thermomechanical indices predict the development of the morphology during injection molding, as suggested in previous works $(19-21,32)$. An increment in the mold temperature results in an increment of the cooling index and a decrease on the thermo-stress index, which may be translated into a higher degree of crystallinity and lower level of molecular orientation of the moldings.

Figure 2 reveals a close coupling between the thermal and mechanical phenomena in the injection molding process. This suggests also a close relationship between the morphological parameters, namely the degree of crystallinity and the level of molecular orientation of the moldings. These dependences are respectively shown in Fig. 12. As expected, a molding with a high level of molecular orientation also shows a low degree of crystallinity, as a result of the strong thermo-mechanical coupling (19-21).

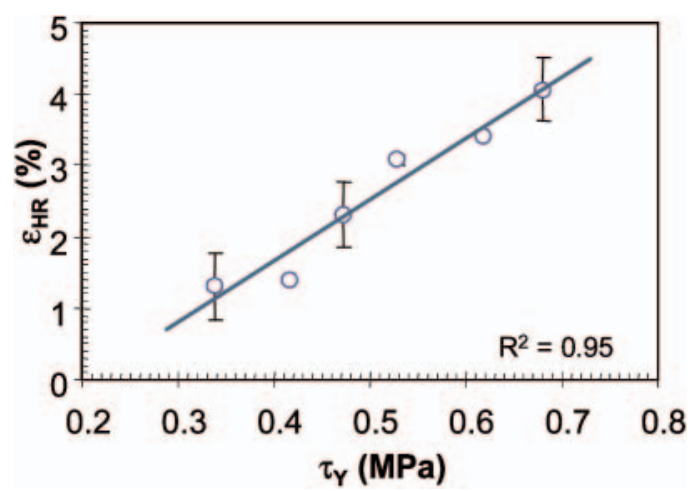

Fig. 11. Variation of the hot recoverable strain, $\varepsilon_{H R}$, with the thermo-stress index, $\tau_{Y}$ (solid line is a linear fit to the data). [Color figure can be viewed in the online issue, which is available at www.interscience.wiley.com.] 


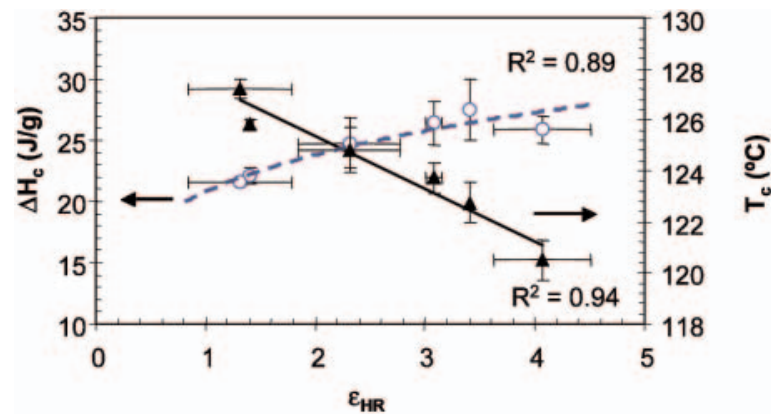

Fig. 12. Dependence between the enthalpy of cold crystallization, $\Delta H_{c}$, and cold crystallization peak temperature, $T_{c}$, upon the hot recoverable strain, $\varepsilon_{H R}$. (solid line is linear fit to the data: $T_{c}=59.39+0.458 \varepsilon_{H R}$; dashed line is power fit to the data: $\Delta H_{c}=20.789 \varepsilon_{H R}{ }^{0.195}$ ). [Color figure can be viewed in the online issue, which is available at www.interscience.wiley. com.]

\section{Morphology-Mechanical Property Relationships}

As mentioned above, the variations in the mechanical properties of the moldings should be related to the morphology developed upon the constraints imposed by the processing thermomechanical environment. Figure 13 shows the evolution of the initial modulus, E, with the cold crystallization enthalpy, $\Delta H_{c}$, and the percentage of hot recoverable strain, $\varepsilon_{\mathrm{HR}}$. The initial modulus increases with $\varepsilon_{\mathrm{HR}}$ and is much less sensitive to variations of $\Delta H_{c}$. This reflects, for this particular case, a strong dependence of $\mathrm{E}$ on the level of amorphous molecular orientation. For these polymers with very low degree of crystallinity, E does not depend upon the degree of crystallinity. This reveals the importance of the amorphous phase for the initial deformation stages. Furthermore, the relative low coefficient of multiple correlation, $R^{2}$, means that other morphological parameters should be considered (e.g., the entanglements density). Also in Fig. 13, the variations of $\mathrm{E}$ fall in a small strip-like region. This is a consequence of the close relationship between the degree of crystallinity and

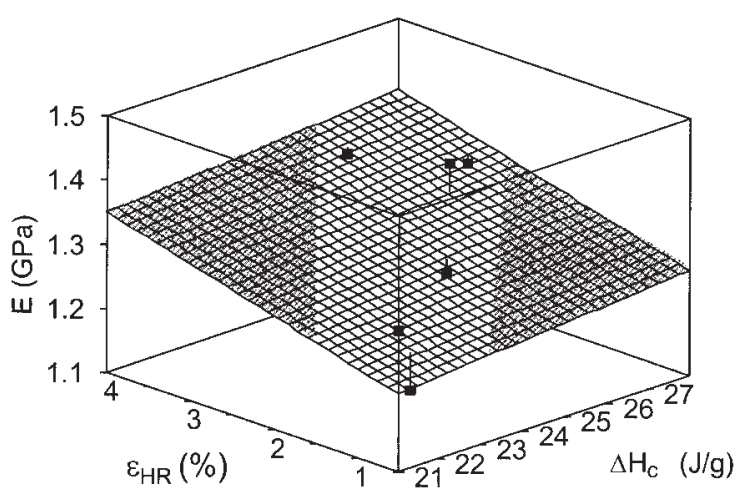

Fig. 13. Variation of the initial modulus, $E$, with the cold crystallization enthalpy, $\Delta H_{c}$, and the hot recoverable strain, $\varepsilon_{H R}$. (the plane is a linear fit to the data: $E=1.066+0.057 \Delta H_{c}+$ $0.036 \varepsilon_{H R} ; R^{2}=0.61$, maximum residual $=4.8 \%$ ). the level of molecular orientation in injection molding, as abovementioned.

Figure 14 shows the evolution of $\sigma_{\mathrm{y}}$ with the cold crystallization enthalpy, $\Delta H_{c}$, and the hot recoverable strain, $\varepsilon_{\mathrm{HR}} \cdot \sigma_{\mathrm{y}}$ increases linearly with the reduction of $\Delta H_{c}$, that is, with the increment in the degree of crystallinity of the moldings. It also increases with the percentage of hot recoverable strain, showing a logarithmiclike trend. It seems that $\sigma_{\mathrm{y}}$ increases with increments in both the degree of crystallinity and the level of molecular orientation. However, the dependence upon the former is higher. Again, as in the case of $\mathrm{E}$, the variations of $\sigma_{\mathrm{y}}$ are in a small region owing to the close coupling between the morphological features.

The evolution of $\varepsilon_{\mathrm{b}}$ with both $\Delta H_{c}$ and $\varepsilon_{\mathrm{HR}}$ does not show good agreement. This means that the high deformation properties of PET could not be satisfactory linked to the initial morphological state (as measured by the evaluated morphological parameters). This may be due to the strong morphological evolution during mechanical drawing (especially at low test velocity) shown by PET $(2,3,33)$. Nevertheless, the general trend (illustrated in Fig. 15) seems to be a substantial reduction of the capability of absorbing energy of the moldings as the level of molecular orientation decreases, and, to a significantly less extent, as the degree of crystallinity decreases. This concomitant diminution of both these morphological features was already observed for the specimens molded with $T_{w}=100^{\circ} \mathrm{C}$ (Fig. 1) and anticipated by the computation of the thermomechanical indices (Fig. 2), resulting in the lowest deformation capabilities of the moldings (Fig. 9).

\section{CONCLUSIONS}

This work studied the relationships between the processing, the morphology and the mechanical properties of a slowly crystallizing and low-crystallinity polymer, PET. The morphology of the polymer was varied through processing by systematic changes on the mold temperature. The morphology development was interpreted by two thermomechanical indices that were related to the

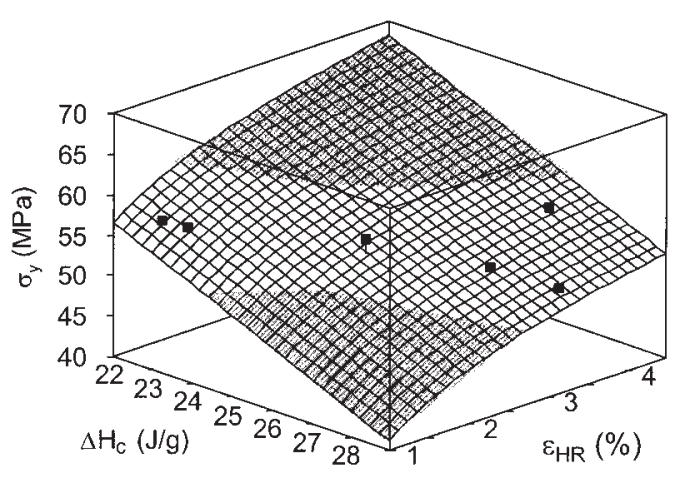

Fig. 14. Variation of the yield stress, $\sigma_{y}$, with the cold crystallization enthalpy, $\Delta H_{c}$, and the percentage of hot recoverable strain, $\varepsilon_{H R}$. (the plane is a fit to the data: $\sigma_{y}=102.48+2.19$ $\Delta H_{c}+7.23 \ln \left(\varepsilon_{H R}\right) ; R^{2}=0.86$, with a maximum residual of $3.2 \%)$. 


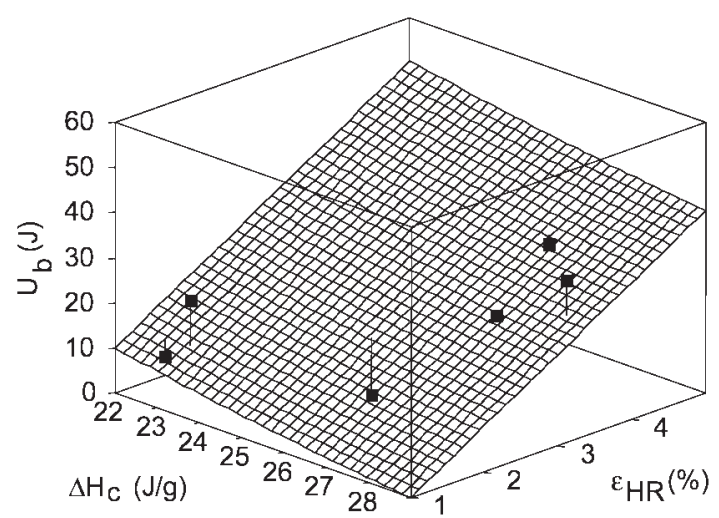

Fig. 15. Variation of the energy at break, $U_{b}$, with the cold crystallization enthalpy, $\Delta H_{c}$, and the percentage of hot recoverable strain, $\varepsilon_{H R}$.

degree of crystallinity and level of orientation of the moldings. The relationships between the morphology and mechanical properties of the moldings were then established. The following conclusions can be drawn.

The mold temperature influences decisively the morphology development in slowly crystallizing PET of very low degree of crystallinity. The cooling index increases with the mold temperature, $T_{w}$, while the thermostress index decreases, anticipating an increment on the degree of crystallinity and a reduction on the level of molecular orientation of the moldings with increasing $T_{w}$. The increment on $T_{w}$ results in an increase on the degree of crystallinity of the moldings (revealed by the reduction of the enthalpy of cold crystallization), and in the reduction of the global level of amorphous molecular orientation (as assessed by the decrease on the percentage of hot recoverable strain). The relationships between the thermomechanical indices and the morphology of the moldings were established: to higher $Y_{c}$ correspond moldings with lower enthalpy of cold crystallization (that is, higher degree of crystallinity); higher $\tau_{Y}$ are related to higher hot recoverable strain values (i.e., high levels of molecular orientation within the amorphous phase). Close relationships between both the thermomechanical indices and the morphological parameters were found, revealing the strong thermal and mechanical coupling in the injection molding process.

The developed morphology definitely determines the mechanical behavior of PET moldings. The initial modulus is mainly dependent upon the level of molecular orientation, showing no variation with the degree of crystallinity. This provides evidence of the role played by the amorphous phase in the initial mechanical deformation stages (although other morphological parameters should also be considered). The yield stress is influenced by both the degree of crystallinity and the level of molecular orientation, but more significantly by the former. The strain at break cannot be satisfactory linked to the initial morphological state, due to the strong morphological transformations expectantly occurring during deformation. Nevertheless, the general trend seems to be a substantial reduction of the deformation capability of the moldings as the level of molecular orientation decreases and, but in a significant less extent, as the degree of crystallinity decreases.

The findings of this work can be quite general and may be extrapolated to other polymeric systems of low crystallization kinetics. From a practical point of view, the results may contribute to some insights for the particular case of the injection molding of PET that is extremely relevant in the production of pre-forms for bottle processing (stretch-blow molding).

\section{ACKNOWLEDGMENTS}

The authors are indebted to Selenis (www.selenis. com) for supplying the PET material. This work was financially supported by FCT-Foundation for Science and Technology, through the POCTI and FEDER programs. The XRD experiments were supported by the IHP Contract HPRI-CT-1999-00040 of European Commission and HASYLAB Project II-01-006 EC.

\section{REFERENCES}

1. K. Schmidt-Rohr, W. Hu, and N. Zumbulyadis, Science, 280, 714 (1998).

2. J. S. Zaroulis and M. C. Boyce, Polymer, 38, 6, 1303 (1997).

3. P. G. Llana and M. C. Boyce, Polymer, 40, 6729, (1999).

4. S. S. Katti and J. M. Shultz, Polym. Eng. Sci., 22, 1001 (1982).

5. M. Fujiyama, in Polypropylene Structure, Blends and Composites: Structure and Morphology, vol. 1, pp. 167-204, J. Karger-Kocsis, ed., Chapman \& Hall, London (1995).

6. Y. Ulcer and M. Cakmak, Polymer, 38, 12, 2907 (1997).

7. Y. Ulcer, M. Cakmak, J. Miao, and C. M. Hsiung, J. Appl. Polym. Sci., 60, 669 (1996).

8. C. M. Hsiung, M. Cakmak, and Y. Ulcer, Polymer, 37, 20, 4555 (1996).

9. N. M. Neves, A. S. Pouzada, J. H. D. Voerman, and P. C. Powell, Polym. Eng. Sci., 38, 1770 (1998).

10. C. Santa Cruz, F. J. Baltá Calleja, H. G. Zachmann, N. Stribeck, and T. J. Asano, J. Polym. Sci., Polym. Phys. Ed., 29, 819 (1991).

11. C. Santa Cruz, N. Stribeck, H. G. Zachmann, and F. J. Baltá Calleja, Macromolecules, 20, 5980 (1991).

12. S. Montserrat, P. Colomer, and J. Belana, J. Mat. Chem., 2, 217 (1992)

13. J. A. Diego, J. C. Cañadas, M. Mudarra, and J. Belana, Polymer, 40, 5355 (1999).

14. N. M. Alves, J. F. Mano, E. Balaguer, J. M. Meseguer Dueñas, and J. L. Gómez Ribelles, Polymer, 43, 4111 (2002).

15. R. Phillips, G. Herbert, J. News, and M. Wolkowicz, Polym. Eng. Sci., 34, 1731 (1994).

16. A. M. Cunha and A. S. Pouzada, in Impact and Dynamic Fracture of Polymers and Composites, Esis 19, pp. 315-325, J. G. Williams and A. Pavan, eds., Mech. Eng. Pub., London (1995).

17. P. Zipper et al., Int. Polym. Proc., XII, 2, 192 (1997).

18. J. C. Viana, A. M. Cunha, and N. Billon, J. Mat. Sci., 36 , 4411 (2001).

19. J. C. Viana, PhD thesis, University of Minho (2000).

20. A. M. Cunha, J. S. Godinho, and J. C. Viana, in Structure Development During Polymer Processing, NATO-ASI series, vol. 370, pp. 255-277, A. M. Cunha and S. Fakirov, eds., Kluwer Academic Pub., Dordrecht, The Netherlands (2000). 
21. J. C. Viana, A. M. Cunha, and N. Billon, Polymer, 43, 4185 (2002).

22. J. F. Agassant, P. Avenas, and J. Ph. Sergent, Polymer Processing, Principles and Modelling, Hanser Gardner Publications, London (1991).

23. M.D. Bullwinkel, S. E. Campbell, G. Nagarajan, and C. Wang, SPE ANTEC Tech. Papers, 42, 615 (1996).

24. Z. Kiflie, S. Piccarolo, V. Brucato, and F. J. Baltá-Calleja, Polymer, 44, 4487 (2002).

25. Z. Zhang, M. Ren, J. Zhao, S. Wu, and H. Sun, Polymer, 44, 2547 (2003).

26. R. Satoto, J. Morikawa, and T. Hashimoto, Polym. Intern., 48, 509 (1999).

27. M. Kattan, E. Dargent, and J. Greet, Polymer, 43, 1399 (2002)
28. D. M. Fann, S. T. Huang, and J. Y. Lee, J. Appl. Polym. Sci., 61, 261 (1996).

29. E. Dargent, J. Grenet, and A. Dahoun, Polym. Eng. Sci., 37, 1853 (1997).

30. C. M. Roland and M. F. Sonnenschein, Polym. Eng. Sci, 31, 1434 (1991).

31. J. C. Viana, A. M. Cunha, and N. Billon, Proc. 18th PPS Conf. (CD-ROM edition), Guimarães (2002).

32. J. C. Viana, A. M. Cunha, and N. Billon, Polym. Eng. Sci. 44, 1522 (2004).

33. E. Gorlier, J.-M. Haudin, and N. Billon, Polymer, 42, 23, 9541 (2001). 